\title{
The effects of artificial selection on genetic variation of some immune genes in Gallus gallus
}

\author{
Tangjie Zhang, Nannan Zhao and Qian Liu \\ College of Veterinary Medicine, Yangzhou University, Yangzhou, China
}

\begin{abstract}
To research effects of the artificial selection of Gallus gallus on $G$. domesticus' nucleotide diversity of immune genes, sequence polymorphisms of $G$. domesticus (23 genes), G. gallus (23 genes), G. lafayetti (17 genes), and G. sonneratii (17 genes) were obtained from GenBank. The data set included 819 polymorphisms. Immune gene polymorphism and selection efficiency in the data from those four species of Gallus were calculated. By calculating the $\mathrm{q}_{\mathrm{w}}$ (Watterson's estimator) of each site, an average $\mathrm{q}_{\mathrm{w}}$ for each species and the minimum number of re-combinations in each species and by estimating the selection efficiency for G. domesticus and $G$. gallus, neither significant nucleotide diversity nor genetic-diversity- $\mathrm{q}_{\mathrm{w}}$ difference was found between $G$. domesticus and $G$. gallus. The results indicated that the patterns of genetic diversity in $G$. domesticus were strongly influenced by recombination and, because Tajima's $D$ has a negative value, recombination was the main mechanism responsible for the immune gene evolution of G. gallus.
\end{abstract}

Keywords: domestication, artificial selection, Gallus gallus, immune gene

\section{Introduction}

Domestic animals have often been artificially selected for certain traits over several thousand years. Poultry domestication is the genetic modification of a wild species to create a new form of a bird to meet human needs. Improvement after domestication has also resulted in striking changes in yield, immune system, biochemical composition and other traits.

The domestic chicken is descended primarily from the Red Junglefowl (G. gallus) in Southeast Asia nearly 10000 years ago (Crawford 1990). But at least one other species

Archiv Tierzucht 56 (2013) 69, 691-699

doi: 10.7482/0003-9438-56-069

Corresponding author:

Tangjie Zhang; email: slx@yzu.edu.cn

College of Veterinary Medicine, Yangzhou University, Yangzhou 225009, China
Received: 07 May 2012

Accepted: 11 June 2013

Online: 14 June 2013

() 2013 by the authors; licensee Leibniz Institute for Farm Animal Biology (FBN), Dummerstorf, Germany. This is an Open Access article distributed under the terms and conditions of the Creative Commons Attribution 3.0 License (http://creativecommons.org/licenses/by/3.0/). 
must have contributed, specifically the grey jungle fowl (Wong et al. 2004), to the domestic chicken. The size, shape and production of the modern domesticated chicken have been sculpted by artificial selection for at least 2000 years, likely contain many important lessons about the genetic architecture of phenotypic variation and the mechanistic basis of selection. Indeed, chicken and other domesticated species played an important role in Darwin's »On the Origin of the Species«, as they provided vivid examples of descent with modification.

Most domesticated animals have experienced »a domestication bottlenec« that reduced genetic diversity relative to their wild ancestor (Buckler 2001). This bottleneck affects all genes in the genome and modifies the distribution of genetic variation among loci. Selection is similar to a more severe bottleneck (Galtier 2000) that removes most of the genetic variation from a target locus. Chicken (G. domesticus) showed a high density of SNP and a high recombination rate, which made it possible to perform high-throughput genotyping to evaluate the existing genetic diversity in chicken at the genome level compared to other species. However, relatively little progress has been made on systematically identifying which immune gene sites of $G$. domesticus genome were influenced by selective breeding during the natural history of chicken.

Here, genetic variations of 23 gene fragments in a sample of Gallus Genus 4 species, $G$. domesticus, G. gallus, G. lafayetii, G. sonneratii on the basis of gene sequence polymorphism were reported and the effects of artificial selection on some immune genes were analysed in G. gallus. The multi-locus analysis is a powerful way to detect adaptation at the population level, so that comparative researches of diversity and recombination in the Gallus genus would help us to comprehensively understand the immune genetic structure and selection in domestication.

\section{Material and methods}

Gallus families sequence polymorphism data set

A total of 819 data sets of Gallus genus gene polymorphisms (1 159 to 9398 base pairs) was obtained from Popset of GenBank and Daniel G. Bradley (2010) including sequence polymorphisms of G. domesticus 23 genes, G. gallus 23 genes, G. lafayetii 17 genes and G. sonneratii 17 genes. Each group was aligned by eye using CLUSTALW (Thompson et al. 1994). Alignments of all groups are available on request.

\section{Polymorphism sequence data analyses}

The average variability $\mathrm{Pi}$ and minimum number of the recombination parameter were calculated by using DnaSP v. 5 (Librado \& Rozas 2009). Insertions/deletions (indels) were excluded from all estimates. To investigate the evidence of the non-neutral evolution, the $D$ test of Tajima was applied (Tajima 1989).

The diversity was measured by Watterson's estimator of the population mutation parameters $\left(q_{w}\right)$, which was calculated separately from the non-synonymous and silent sites for the con-specific gene fragments. The parameter represents the per-site diversity. 


$$
q_{w}=\frac{P}{L \sum_{i=1}^{n-1} \frac{1}{i}}
$$

$P$ is the number of synonymous polymorphisms, $L$ is the number of synonymous sites and $n$ is the number of the sequence sampled.

We calculated the efficiency of selection for four species. Measurement of selection efficiency is as following:

$$
\frac{q_{n}}{q_{s+i}}=\frac{\sum_{s} P_{n} / \sum_{n} L_{n}}{\left(\sum_{s} P_{i}+\sum_{i} P_{i}+1\right) /\left(\sum_{s} L_{s}+\sum_{i} L_{i}\right)}
$$

where $P_{n^{\prime}} P_{s}$ and $P_{i}$ are the numbers of non-synonymous, synonymous and intron polymorphisms. $L_{n^{\prime}} L_{s}$ and $L_{i}$ are the numbers of non-synonymous, synonymous and intron sites for each gene in each species. $q_{n}$ is for non-synonymous sites. $q_{s+i}$ is for synonymous and intron correspondingly. $q$ were arc-sine transformed. Recombination parameter was log $x+1$ transformed. After the calculation of $q_{s}$ for synonymous sites and $q_{i}$ for intron sites, the weighted average of $q_{s}$ or/and $q_{i}$ from different genes for same species was made.

\section{Results}

Whole sequence segment variations in G. domesticus and G. gallus

More than $84 \mathrm{~kb}$ of the DNA sequence was obtained across 23 immune genes. Sequence data for the CR1 and OTC gene were obtained from only G. domesticus and G. gallus. Summary statistics of the number of segregating sites, nucleotide diversity $\mathrm{Pi}, \mathrm{q}_{\mathrm{w}^{\prime}}$, Tajima's $\mathrm{D}$ test and the minimum number of recombination events were shown in Table 1. Single variable regressions of immune gene nucleotide diversity showed that no significant difference was found between $G$. domesticus and $G$. gallus $\left(F_{2,23}=1.39, P=0.5845>0.05\right)$. Neither did the diversity- $q_{w}$ of the fragments $\left(F_{2,22}=0.074, P=0.7866>0.05\right)$.

The population recombination parameter, $\rho$, is the other key parameter in simple population genetic models. However, the estimation of $\rho$ requires considerably larger segments of contiguous DNA to be sequenced (Hudson 2001). The relatively short sequences obtained in this study are not sufficient to provide reliable locus-specific estimates of $\rho$. Instead, the estimation of the minimum number of the recombination parameter was obtained from the two sample species. Single variable regressions revealed that the recombination parameter of $G$. domesticus was significantly higher than that of $G$. gallus $\left(F_{2,23}=6.160, P=0.0169<0.05\right)$ after recombination parameter was log $\mathrm{x}+1$ transformed. By using DnaSP, recombination parameter was estimated, which is inversely proportional to $L D$ (linkage disequilibrium). The average of estimates of recombination parameter in G. domesticus is $164 \%$ of that in G. gallus, while the average of estimates of $\mathrm{q}_{w}$ in $G$. domesticus is $95 \%$ of that in $G$. gallus (Figure 1). Thus, the recombination parameter in $G$. domesticus has been reduced more drastically than the population mutation parameter $\mathrm{q}_{\mathrm{w}^{\prime}}$ contrary to what has been expected under a population 
Table 1

$\mathrm{Pi}$, Theta, TajimaD and recombination parameter of 23 gene sequence for 4 species

\begin{tabular}{|c|c|c|c|c|c|c|c|c|c|c|c|c|c|}
\hline Gene & $\mathrm{L}$ & $\begin{array}{l}\text { Ns } \\
\text { G. } d .\end{array}$ & $\begin{array}{l}\text { Ns } \\
\text { G. } g .\end{array}$ & $\begin{array}{c}\mathrm{N} \\
\text { G. } d .\end{array}$ & $\begin{array}{c}\mathrm{N} \\
\text { G. } g .\end{array}$ & $\begin{array}{c}\mathrm{Pi} \\
\text { G. d. }\end{array}$ & $\begin{array}{c}\mathrm{Pi} \\
\text { G. } g .\end{array}$ & $\begin{array}{c}\mathrm{q}_{\mathrm{w}} \\
\text { G.d. }\end{array}$ & $\begin{array}{c}\mathrm{q}_{\mathrm{w}} \\
G . g .\end{array}$ & $\begin{array}{c}\text { D } \\
\text { G. } d .\end{array}$ & $\begin{array}{c}\text { D } \\
\text { G. } g .\end{array}$ & $\begin{array}{l}\mathrm{Rm} \\
\text { G. } d .\end{array}$ & $\begin{array}{l}\mathrm{Rm} \\
\text { G. } g .\end{array}$ \\
\hline KK34 & 4381 & 17 & 10 & 26 & 8 & 0.001126 & 0.000858 & 0.001019 & 0.00088 & 0.3702 & -0.137 & 5 & 1 \\
\hline TLR15 & 3105 & 21 & 20 & 21 & 8 & 0.001886 & 0.002059 & 0.001855 & 0.00248 & 0.0625 & -0.8914 & 9 & 4 \\
\hline TLR1LA & 3559 & 41 & 13 & 26 & 8 & 0.00168 & 0.001726 & 0.003019 & 0.00141 & -1.679 & 1.1403 & 4 & 2 \\
\hline TLR1LB1 & 6047 & 80 & 52 & 30 & 8 & 0.0032 & 0.003874 & 0.003339 & 0.00332 & -0.1587 & 0.9058 & 33 & 20 \\
\hline TLR1LB2 & 9398 & 41 & 4 & 30 & 8 & 0.000692 & 0.000179 & 0.001101 & 0.00016 & -1.3716 & 0.3842 & 5 & 0 \\
\hline TLR2B & 4186 & 4 & 7 & 30 & 8 & 0.000328 & 0.000687 & 0.000266 & 0.00071 & 0.6009 & -0.1632 & 1 & 0 \\
\hline TLR3 & 6628 & 123 & 70 & 30 & 8 & 0.004168 & 0.004117 & 0.004684 & 0.00407 & -0.4229 & 0.0579 & 41 & 19 \\
\hline TLR4 & 6237 & 58 & 46 & 30 & 8 & 0.002611 & 0.002938 & 0.002347 & 0.00284 & 0.4222 & 0.1757 & 17 & 16 \\
\hline TLR5 & 3150 & 59 & 8 & 19 & 2 & 0.002696 & 0.00254 & 0.005359 & 0.00254 & $-2.035^{* *}$ & na & 4 & 0 \\
\hline TLR7 & 6746 & 71 & 34 & 30 & 8 & 0.001989 & 0.001853 & 0.002657 & 0.00194 & -0.9503 & -0.2489 & 13 & 8 \\
\hline GMCSF & 2730 & 15 & 16 & 30 & 8 & 0.001929 & 0.002272 & 0.001392 & 0.00227 & 1.2985 & 0.0065 & 3 & 4 \\
\hline IL13 & 2794 & 38 & 34 & 27 & 8 & 0.004942 & 0.004883 & 0.003529 & 0.00469 & 1.4994 & 0.2151 & 14 & 0 \\
\hline IL12A & 2005 & 33 & 24 & 30 & 8 & 0.004446 & 0.005825 & 0.004155 & 0.00462 & 0.2556 & 1.3747 & 12 & 4 \\
\hline IL9 & 2954 & 57 & 44 & 26 & 8 & 0.003885 & 0.005356 & 0.005057 & 0.00575 & -0.8904 & -0.3629 & 15 & 10 \\
\hline IL8 & 3114 & 32 & 19 & 28 & 8 & 0.002309 & 0.002523 & 0.002641 & 0.00235 & -0.4617 & 0.375 & 7 & 4 \\
\hline IL5 & 4686 & 34 & 20 & 30 & 8 & 0.002423 & 0.002506 & 0.002712 & 0.00244 & -0.389 & 0.1452 & 8 & 1 \\
\hline IL4 & 1998 & 17 & 21 & 30 & 8 & 0.003122 & 0.00395 & 0.002148 & 0.00405 & 1.5513 & -0.133 & 10 & 3 \\
\hline IL3 & 5505 & 42 & 29 & 30 & 8 & 0.002675 & 0.001787 & 0.001929 & 0.00204 & 1.4267 & -0.6452 & 19 & 6 \\
\hline CR1_1 & 458 & 2 & 2 & 3 & 3 & 0.002911 & 0.002911 & 0.002911 & 0.00291 & na & na & 0 & 0 \\
\hline CR1_2 & 499 & 9 & 6 & 5 & 3 & 0.008818 & 0.008016 & 0.008657 & 0.00802 & 0.1316 & na & 3 & 0 \\
\hline CR1_3 & 600 & 6 & 12 & 4 & 5 & 0.005 & 0.008333 & 0.005455 & 0.0096 & -0.8086 & -0.9543 & 0 & 0 \\
\hline CR1_4 & 1023 & 7 & 5 & 6 & 2 & 0.003739 & 0.00353 & 0.003244 & na & 0.8878 & na & 1 & 0 \\
\hline OTC & 2210 & 24 & 34 & 6 & 6 & 0.004988 & 0.006345 & 0.004884 & 0.00577 & 0.1331 & -0.4086 & 2 & 4 \\
\hline Mean & & & & & & 0.003 & 0.003 & 0.00323 & 0.0034 & -0.023 & 0.036 & & \\
\hline
\end{tabular}

G. d.: Gallus domestiucs, G. g.: Gallus gallus, L: length of gene, Ns: number of segregating sites, D: Tajima's D, Rm: recombination parameter, na: not available 
Table 2

Different sites' $q_{w}$, an average $q_{w}$ and efficiency of selection for 4 species

\begin{tabular}{|c|c|c|c|c|c|c|c|c|c|c|c|c|}
\hline \multirow[b]{2}{*}{ Gene } & \multicolumn{3}{|c|}{ G. domesticus } & \multicolumn{3}{|c|}{ G. gallus } & \multicolumn{3}{|c|}{ G. lafayetii } & \multicolumn{3}{|c|}{ G. sonneratii } \\
\hline & $q_{s}$ & $q_{n}$ & $q_{s+i}$ & $q_{s}$ & $q_{n}$ & $q_{s+i}$ & $q_{s}$ & $\mathrm{q}_{\mathrm{n}}$ & $q_{s+i}$ & $q_{s}$ & $q_{n}$ & $q_{s+i}$ \\
\hline KK34 & 0.01184 & 0.00331 & 0.00079 & 0.014 & 0.00097 & 0.00087 & 0 & 0 & 0.00027 & 0 & 0.00137 & 0.00027 \\
\hline TLR15 & 0.00459 & 0.00109 & 0.00325 & 0.0052 & 0.00124 & 0.00355 & 0.003 & 0.00081 & 0.00199 & 0.0091 & 0.00217 & 0.00897 \\
\hline TLR1LA & 0.00234 & 0.00263 & 0.00226 & 0.0014 & 0.00102 & 0.00186 & & & & 0 & 0.00058 & 0.00066 \\
\hline TLR1LB1 & 0.00171 & 0.00183 & 0.00384 & 0.0026 & 0.00153 & 0.00392 & 0 & 0 & 0.00066 & 0 & 0.00066 & 0.00221 \\
\hline TLR1LB2 & 0.00296 & 0.001 & 0.00109 & 0 & 0.00084 & 0 & 0 & 0.00059 & 0.00014 & 0.0019 & 0.00108 & 0.00013 \\
\hline TLR2B & 0.00051 & 0.00032 & 0.00033 & 0.0008 & 0.00023 & 0.00111 & 0.001 & 0 & 0.00078 & 0.002 & 0.00059 & 0.00239 \\
\hline TLR3 & 0.00629 & 0.00291 & 0.00441 & 0.0062 & 0.00374 & 0.00416 & 0.006 & 0.0025 & 0.00433 & 0.0059 & 0.00166 & 0.00449 \\
\hline TLR4 & 0.00511 & 0.00117 & 0.00288 & 0.0072 & 0.00119 & 0.00359 & 0.01 & 0.00169 & 0.00266 & 0.0083 & 0.00113 & 0.00292 \\
\hline TLR5 & 0.00462 & 0.00121 & 0.0055 & 0.0051 & 0.002 & 0.00348 & 0.016 & 0.00464 & 0.01374 & & & \\
\hline TLR7 & 0 & 0 & 0.00418 & 0 & 0.00016 & 0.00297 & 0.001 & 0.00041 & 0.0028 & 0.0008 & 0.00022 & 0.00572 \\
\hline GMCSF & 0.00245 & 0.00077 & 0.00148 & 0.0075 & 0 & 0.00258 & 0.005 & 0 & 0.00251 & 0 & 0 & 0.0016 \\
\hline IL13 & 0.00738 & 0.00083 & 0.00387 & 0.011 & 0.00124 & 0.00513 & 0 & 0 & 0.00439 & 0.0052 & 0 & 0.00352 \\
\hline IL12A & 0.00225 & 0.00304 & 0.00437 & 0.0034 & 0 & 0.00553 & 0 & 0 & 0.00065 & 0.0097 & 0.00329 & 0.00815 \\
\hline IL9 & 0.00553 & 0.00163 & 0.00548 & 0.0041 & 0 & 0.00645 & 0 & 0 & 0.00608 & 0 & 0 & 0.00995 \\
\hline IL8 & 0 & 0 & 0.00286 & 0 & 0 & 0.00255 & 0 & 0 & 0.00095 & 0.0071 & 0 & 0.00455 \\
\hline IL5 & 0.00543 & 0.0037 & 0.00267 & 0 & 0 & 0.00255 & 0 & 0 & 0.00216 & 0 & 0 & 0.00162 \\
\hline IL4 & 0.00265 & 0.00084 & 0.00233 & 0.0039 & 0.0037 & 0.00412 & 0 & 0 & 0.00259 & 0 & 0.00175 & 0.00194 \\
\hline IL3 & 0 & 0 & 0.00205 & 0 & 0 & 0.00219 & 0 & 0 & 0.00074 & 0 & 0 & 0.00074 \\
\hline CR1_1 & & & 0.00291 & & & 0.00291 & & & & & & \\
\hline CR1_2 & & & 0.00866 & & & 0.00802 & & & & & & \\
\hline CR1_3 & & & 0.00545 & & & 0.0096 & & & & & & \\
\hline CR1_4 & 0.00367 & 0.0031 & 0.00367 & 0.0056 & 0.00283 & 0.0056 & & & & & & \\
\hline OTC & & 0.00488 & & & 0.00577 & & & & & & & \\
\hline Weighted & & 0.00173 & 0.00287 & & 0.00171 & 0.00293 & & 0.00117 & 0.00216 & & 0.00094 & 0.00290 \\
\hline $\mathrm{q}_{\mathrm{n}} / \mathrm{q}_{\mathrm{w}}$ & & 0.3760 & & & 0.3685 & & & 0.3514 & & & 0.2448 & \\
\hline $\mathrm{q}_{n} / \mathrm{q}_{s+i}$ & & 0.60192 & & & 0.58515 & & & 0.54188 & & & 0.32559 & \\
\hline
\end{tabular}



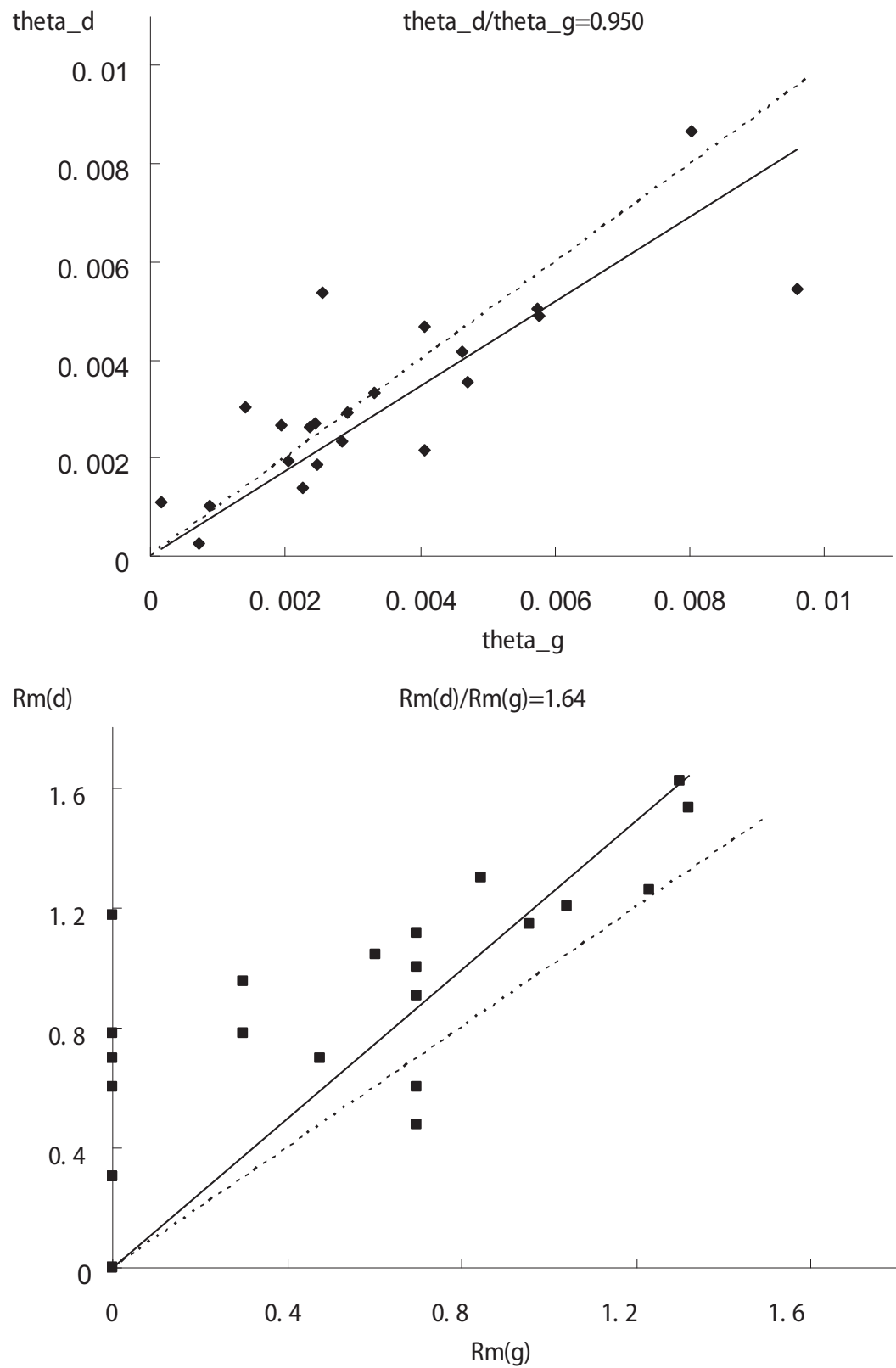

The first row illustrates the relationship between mean values of $\mathrm{q}_{w}$ in $G$. domesticus (y-axis) versus G. gallus ( $x$-axis). Dashed diagonal lines have a slope of 1.0, representing equal diversity between taxa; solid lines lines are regression lines. Each square represents a single gene.

The second row plots the relationship between estimates of minimum number of recombination in $G$. domesticus (y-axis) versus G. gallus (x-axis).

Figure 1

Patterns of diversity in G. domesticus and G. gallus at 23 gene fragments 
bottleneck (Wall et al. 2002). The results suggest that patterns of immune gene diversity in $G$. domesticus are strongly influenced by recombination.

Tajima's D test is to distinguish between a DNA sequence evolving neutrally and DNA evolving under a non-random process, including selection, demographic expansion or contraction. In order to perform the test, homologous DNA for at least three individuals was required, so that five sequence polymorphism groups were not available (Table 1). In terms of 23 sampled genes, TLR5 in G. domesticus was found to be statistically significant for Tajima's D $(P<0.05)$. In principle, this could potentially indicate a deviation from neutrality, possibly due to strong selection. An average negative Tajima's D in G. domesticus ( -0.023$)$, in contrast to $G$. gallus (0.036), signifies slightly more low frequency polymorphisms, indicating a population size expansion and/or selection in G. domesticus.

\section{Variation of different sites of gene sequence and efficiency of selection in Gallus genus}

Across the 23 genes, 84013 base pairs were aligned, including 831 mutation sites. The comparison of different sites' $\mathrm{q}_{w^{\prime}}$ a weighted average of $\mathrm{q}_{\mathrm{w}}$ and selection efficiency for four species was shown in Table 2 . The weighted average of $q_{n}$ for non-synonymous sites was arranged as follows: $G$. domesticus $(0.00173)$, G. gallus $(0.00171), G$. lafayetii $(0.00117)$ and G. sonneratii (0.00094). In the top-to-bottom order, while the difference of the weighted average $\mathrm{q}_{\mathrm{s}+\mathrm{i}}$ for four species was not found, the arrangement was as follows: $G$. domesticus (0.00287), G. gallus (0.00293), G. lafayetii (0.00216) and G. sonneratii (0.00290), respectively. $\mathrm{q}_{\mathrm{n}} / \mathrm{q}_{\mathrm{w}}$ represented the percent of mutations of the non-synonymous in total gene segment mutations. G. domesticus showed the highest value of $q_{n} / q_{w}(0.60192)$ for four species analysed and suggested that $G$. domesticus' immune genes may undergo more strong pressure of selection.

\section{Discussion}

In this paper, sequence segment variations, diverse sites weighted mutations $\left(q_{w}\right)$, recombination parameter and efficiency of selection within Gallus genus were determined. The results demonstrated the artificial selection of $G$. domesticus and indistinguishable immune genetic diversity with three other species.

A diversity index $(\mathrm{Pi})$ analysis indicated that the overall nucleotide variability of all 23 immune genes for $G$. domesticus and G. gallus were approximately 0.003 and showed no difference, as mentioned above. The population recombination rate $\rho$ is a fundamental parameter for evolutionary biology. Not only recombination is a key force shaping the architecture of genomes, but also distribution across genomic regions is essential for association studies of traits. However, the estimation of the population recombination rate is not an easy task. Adequate and reliable locus-specific estimates of $\rho$ could not be provided with relatively short sequences, as we failed in estimation by LDhat v2.0 (McVean 2004), a package for the analysis of recombination rates from population genetic data. We turned to calculate the minimum number of the recombination parameter. The result revealed that the recombination parameter in $G$. domesticus was higher than that in G. gallus in most of the sampled loci (21/23). 
Of 84013 base pairs, 831 mutation sites were found. This is somewhat more than the extensive sequence diversity present in domestic chicken ( $\sim 5$ single nucleotide polymorphisms per kilobase in pairwise comparisons) (Wong et al. 2004), mainly as a result of the sample size.

Regions of intergenic, noncoding DNA where levels of variation are expected to be higher (Zwick et al. 2000) may provide a different picture of diversity. Our estimates of the weighted average of $q_{w}$ for both silent mutation sites and non-synonymous sites indicated that $q_{w}$ for silent mutation sites in total species were higher than that for non-synonymous sites, as we expected. G. lafayetii has the lowest synonymous mutation $\mathrm{q}_{\mathrm{w}}=0.00216$ among four species, which is obviously correlated to its effective population size.

The mutation parameter of non-synonymous sites- $\mathrm{q}_{\mathrm{n}}$-and percent of non-synonymous mutations in total segment mutations- $\mathrm{q}_{\mathrm{n}} / \mathrm{q}_{\mathrm{w}}$-for $\mathrm{G}$. domesticus and $\mathrm{G}$. gallus were higher than that for G. lafayetii and $G$. sonneratii, which indicated that the immune genes of $G$. domesticus and $G$. gallus could undergo a stronger directional selection pressure. This was inconsistent with previous researches that selection for body weight in chicken has depressed immune performance (Miller et al. 1992) and antibody production (Cheema et al. 2003). Now that almost equivalent $\mathrm{q}_{\mathrm{w}}$ for $\mathrm{G}$. domesticus and $G$. gallus, there should be some factors which could decrease nuclear diversity of $G$. domesticus since this species obviously have high effective population size, Ne. Of these factors, high recombination event in $G$. domesticus was inferred to be essential.

The highest efficiency of selection (0.60192) was found in G. domesticus (Table 2). This selection was mainly described as artificial selection for the needs of human being here, which could be confirmed by negative noticeable Tajima's $D$.

A population bottleneck was not found by analysis of mutation and recombination parameter. Nevertheless, G. domesticus did experience severe population bottleneck (Mason 1984) although this bottleneck effect did not result in a substantial loss of genetic diversity. Abroad crossing between breeds and higher recombination could be fundamental explanations for undifferentiated diversity between $G$. domesticus and G. gallus.

Of particular interest will be to define the number of loci responsible for shaping the diversity of form and function, the types of genes and genetic variation therein that have responded to artificial selection. Although our results did not provide definitive answers to these issues, they did afford some insight into the mechanistic basis of artificial selection. Despite the insights gleaned from our data, one limitation of this study was that it did not provide information about more gene sequence polymorphisms. The difference in part reflected differences in sampling.

\section{Acknowledgements}

This research was supported by the Priority Academic Program Development of Jiangsu Higher Education Institutions and Jiangsu Co-innovation Center for Prevention and Control of important Animal Infectious Diseases and Zoonoses, Yangzhou, 225009, China. 


\section{References}

Buckler IV ES, Thornsberry JM, Kresovich S (2001) Molecular Diversity, Structure and Domestication of Grasses. Genet Res 77, 213-218

Cheema MA, Qureshi MA, Havenstein GB (2003) A comparison of the immune response of a 2001 commercial broiler with a 1957 randombred broiler strain when fed representative 1957 and 2001 broiler diets. Poult Sci 82, 1519-1529

Crawford RD (1990) Origin and history of poultry species. In: Crawford RD (ed.) Poultry Breeding and Genetics. Elsevier, Amsterdam, the Netherlands, 1-41

Darwin CR (1872) The Origin of Species. 6th ed. Murray, London, UK

Galtier N, Depaulis F, Barton NH (2000) Detecting Bottlenecks and Selective Sweeps From DNA Sequence Polymorphism. Genetics 155, 981-987

Hillier LW, et al. (2004) Sequence and comparative analysis of the chicken genome provide unique perspectives on vertebrate evolution. Nature 432:695-716

Hudson RR (2001) Two-Locus Sampling Distributions and Their Application. Genetics 159, 1805-1817

Librado P, Rozas J (2009) DnaSP v5: a software for comprehensive analysis of DNA polymorphism data. Bioinformatics 25: 1451-1452

Mason IL (1984) Evolution of domesticated animals. Longman, London et al., UK

McVean G. (2004) LDhat: A package for the population genetic analysis of recombination. Oxford, UK

Miller LL, Siegel PB, Dunnington EA (1992) Inheritance of Antibody Response to Sheep Erythrocytes in Lines of Chickens Divergently Selected for Fifty-Six-Day Body Weight and Their Crosses. Poult Sci 71, 47-52

Tajima F (1989) Statistical method for testing the neutral mutation hypothesis by DNA polymorphism. Genetics 123, 585-595

Thompson JD, Higgins DG, Gibson TJ (1994) CLUSTAL W: improving the sensitivity of progressive multiple sequence alignment through sequence weighting, position-specific gap penalties and weight matrix choice. Nucl Acids Res 22 , 4673-4680

Wall JD, Andolfatto P, Przeworski M (2002) Testing Models of Selection and Demography in Drosophila simulans. Genetics 162, 203-216

Wong GKS et al. (2004) A genetic variation map for chicken with 2.8 million single-nucleotide polymorphisms. Nature 432, 717-722

Zwick ME, Cutler DJ, Chakravarti A (2000) Patterns of Genetic Variation in Mendelian and Complex Traits. Annu Rev Genomics Hum Genet 1, 387-407 